\title{
Editorial.
}

\section{Inteligencia artificial para la toma de decisiones}

\section{JULIÁN ANDRÉS ZAPATA CORTÉS*}

Los administradores y gerentes deben enfrentar procesos complejos de toma de decisiones con el fin de obtener resultados positivos en el entorno. Procesos caracterizados por la alta competencia empresarial, volatilidad de mercados y ambientes cambiantes; estos - en muchos casos- implican un alto número de variables, información, alternativas de decisión y velocidad de respuesta que hacen que tomar una decisión acertada se vuelva casi imposible sin el uso de técnicas o herramientas que les ayude en esta labor.

Los procesos de toma de decisión muchas veces se realizan con base en experiencias, conocimientos, vivencias y anécdotas entre otras situaciones de la vida que se han encargado de forjar el criterio decisivo del tomador de decisiones; y aunque estos elementos son fundamentales para un correcto proceso decisivo también son un arma de doble filo al poder sesgar las decisiones hacia el mismo tipo de soluciones, lo que impide florecer nuevas opciones que puedan generar mejores resultados. En este sentido es posible que las decisiones que se basan únicamente en experiencias y en juicios de los tomadores de decisiones, produzcan siempre las mismas respuestas y de esta manera las mismas soluciones a los problemas (Zapata Cortés, Arango Serna and Moreno Martínez, 2012).

Estos procesos de toma de decisión — que además de ser complejos son una actividad critica para el éxito de las empresas- deben tener en cuenta la experiencia de las personas involucradas, altos volúmenes de información disponible en la empresa y en el entorno y la aplicación de herramientas tanto cualitativas como cuantitativas que les permitan asegurar la calidad de las decisiones y realizar estos procesos en el tiempo requerido (Hernández y Rodríguez, 2006; Chiavenato, 2006; Zapata, Arango y Adarme, 2010).
COMO CITAR ESTE ARTÍCULO

How to cite this article:

Zapata Cortés, J.A. (2020). Editorial. Inteligencia artificial para la toma de decisiones. Revista Perspectiva Empresarial, 7(2-1), 3-5.

\footnotetext{
* PhD en Ingeniería-Industria y Organizaciones. Editor Institucional, Fundación Universitaria CEIPA, Sabaneta, Colombia. E-mail: julian.zapata@ceipa.edu.co. ORCID: 0000-0002-1270-3577. Google Scholar: https://scholar.google.com/citations?user=j2CV8VYAAAAJ\&hl=en.
} 
El mundo de hoy, caracterizado por las nuevas tecnologías de la información, ofrece a los gerentes y administradores la posibilidad de contar con herramientas informáticas para mejorar los procesos de toma de decisión y técnicas de análisis avanzadas para el procesamiento y análisis de la información; con ello es posible realizar procesos de toma de decisiones más acertados y ágiles con capacidad de ser utilizados en procesos complejos que involucren no solo un alto número de variables, sino también múltiples criterios y personas.

Estos sistemas ayudan a superar las deficiencias cognitivas de los tomadores de decisión para integrar múltiples fuentes y tipos de información, generar un proceso de estructuración para el proceso de decisión, basar las respuestas en alternativas claramente definidas y utilizar técnicas formales de diferentes ramas de la ciencia tales como la ingeniería, economía, investigación de operaciones, estadística y los sistemas (Zapata Cortés, Arango Serna and Moreno Martínez, 2012).

La familia de las herramientas informáticas que ayudan a la toma de decisiones se conoce con el nombre de sistemas de soporte a las decisiones -DSS - (Decision Support System) y se encargan de realizar procesos de modelización de la información y posibles situaciones; con ello es posible predecir el resultado de las acciones a emprender o la alternativa que mejor se acomode a la organización, permitiendo con esto decisiones más acertadas (Arnott and Pervan, 2005). Estos sistemas pueden obtener y procesar información proveniente de diferentes fuentes, siendo posible utilizar datos sin organizary con múltiples formatos (como, por ejemplo, documentos de texto, hojas de cálculo, el conocimiento de las personas), lo que se convierte en la entrada para el proceso decisional. Una vez la información es obtenida por el sistema de información su procesamiento se puede realizar básicamente de tres maneras, a saber: análisis de toma de decisiones pasivos, activos y cooperativos.

Los análisis pasivos únicamente recolectan y organizan la información para que el tomador de decisiones la analice por su cuenta y a partir de allí defina la solución que más le satisfaga, por lo que la pasividad de este tipo de sistemas se deriva de que no establecen ninguna sugerencia de respuesta o decisión a las personas encargadas de esta labor. Por su parte los sistemas activos definen una o varias alternativas de solución al problema a partir de las cuales la persona responsable define la decisión a llevar a cabo, por lo que estos sistemas actúan de manera activa en el proceso de decisiones. Mientras que los sistemas cooperativos recogen lo mejor de estos dos tipos y para ello se encargan de obtener, procesar y analizar la información y con base en esto generar un reporte a los tomadores de decisión que deben revisarla y retroalimentar el sistema de forma que la decisión se tome en conjunto entre el software y las personas, lo que le da el nombre a este tipo de sistemas como colaborativos (Zapata Cortés, Arango Serna and Moreno Martínez, 2012).

Dentro de las posibilidades de mayor relevancia para los sistemas de soporte a la tomas de decisiones se encuentran las técnicas de inteligencia artificial; estas mejoran los procesos mediante la coordinación en la entrega de información, el análisis y la definición de tendencias de grandes volúmenes de datos, ofrecen pronósticos, generan información consistente, analizan incertidumbre, prevén las necesidades de los usuarios y entregan información a los tomadores de decisión de formas más apropiadas y sugieren cursos de acción (Phillips-Wren and Jain, 2006).

Los cambios generados en la ciencia de la computación y su integración con áreas como, por ejemplo, la psicología, la neurociencia y el big data, están revolucionando los procesos de toma de decisión en todos los campos en los que los seres humanos deben tomar decisiones tales como el gubernamental, los negocios, la política y los deportes; a su vez se integran con tecnologías de automatización que inclusive permiten que todo el proceso decisional y de implementación pueda ser llevado a cabo por estas herramientas inteligentes artificialmente (Dear, 2019).

Las técnicas de inteligencia artificial cubren la automatización de tareas cognitivas y físicas. Estas técnicas ayudan a las personas a realizar tareas y tomar decisiones más rápido y mejor. Permite la automatización de la toma de decisiones sin intervención humana, reduciendo así el trabajo humano intensivo y las tareas tediosas. Hay muchas más formas en las que la inteligencia artificial está marcando la diferencia para las empresas como son, por mencionar solo algunas, la toma de decisiones de marketing, la gestión de relaciones con el cliente, los sistemas de recomendación, la resolución de problemas, la minería de opiniones y la analítica aumentada. 
Como ejemplo de lo anterior, en mercadeo es necesario comprender las necesidades y deseos del cliente yalinear los productos con esas necesidadesy deseos. Controlar el cambio de comportamiento del cliente es vital para tomar las mejores decisiones de marketing. Las técnicas de simulación y modelado de inteligencia artificial brindan información confiable sobre la personalidad de los consumidores. Esto ayudará a predecir el comportamiento de los mismos. Mediante la recopilación de datos en tiempo real, el análisis de tendencias y la previsión, un sistema de inteligencia artificial puede ayudar a las empresas a tomar decisiones de marketing interesantes. De igual manera las organizaciones pueden identificar el ciclo de vida de un consumidor con la ayuda de un modelo de personalización del comprador. Durante un proceso complejo de toma de decisiones, la Inteligencia artificial puede gestionar y controlar de forma eficiente diferentes factores al mismo tiempo. Puede obtenery procesar grandes cantidades de datos en cuestión de minutos al tiempo que proporciona información valiosa para la empresa. Además las técnicas de inteligencia artificial han brindado a las empresas información invaluable sobre los consumidores, lo que les ayuda a mejorar su comunicación con ellos. También ayuda a los minoristas a predecir la demanda de productos y responder rápidamente a ella.

De esta manera es fundamental que en el contexto latinoamericano se desarrolle con intensidad el estudio de la inteligencia artificial y las herramientas de toma de decisiones, que permita a los administradores y gerentes tomar mejores decisiones tanto en calidad como en tiempo, de modo que potencialicen la competitividad y el éxito de sus organizaciones.

Es en este sentido que se realiza el presente volumen especial sobre inteligencia artificial de la Revista Perspectiva Empresarial, el cual recoge un grupo de artículos seleccionados del " $1^{\text {th }}$ International Workshop on Enterprise DecisionMaking Applying Artificial Intelligence Techniques (WEDMAIT 2020)"; una apuesta entre varias instituciones colombianas y una mexicana para generar un espacio de socialización y discusión de los trabajos realizados por instituciones educativas y comerciales en las que se pueda evidenciar la aplicación de la inteligencia artificial para mejorar la toma de decisiones de las organizaciones de la región; ello, como un primer paso para lograr la apropiación de estas herramientas e impulsar así el desarrollo competitivo de la región.

\section{Referencias}

Arnott, D. and Pervan, G. (2005). A critical analysis of decision support systems research. Journal of Information Technology, 29(4), 67-87.

Chiavenato, I. (2006). Introducción a la teoría general de la administración. Ciudad de México, México: McGraw-Hill.

Dear, K. (2019). Artificial Intelligence and DecisionMaking. The RUSI Journal, 164(5-6), 18-25.

Hernández y Rodríguez, S. (2006). Introducción a la administración. Ciudad de México, México: McGraw-Hill.

Phillips-Wren, G. and Jain, L. (2006). Artificial Intelligence for Decision Making. En Gabrys, B., Howlett, R.J. and Jain, L.C. (Eds.), KnowledgeBased Intelligent Information and Engineering Systems. Berlin, Heidelberg: Springer.

Zapata, J.A., Arango, M.D. y Adarme, W. (2010). Herramientas tecnológicas al servicio de la gestión empresarial. Revista Avances en Sistemas e Informática, 7(3), 87-101.

Zapata Cortés, J.A., Arango Serna, M. and Moreno Martínez, S. (2012). Expertchoice(C) decision support system implementation for vehicle selection in a Colombian shipyard. Dyna, 79(173), 53-60. 